care. Topics ranged from: symptom management, oncological emergencies, staff wellbeing, nutrition, catheter care, pressure ulcer management, safe use of equipment, learning disabilities and wheelchair training, to mention just a few.

Over time members of the team have taken turns delivering sessions, particularly those who undertake additional link worker roles. Staff have been encouraged to share skills and knowledge gained while attending external study days. The sessions have been running for a year and have evolved during this time. We have reviewed and adapted the sessions to meet the challenges we have encountered.

Colleagues from the multi-disciplinary team, such as our physiotherapist and social worker have undertaken education pertinent to their roles. This has resulted in better understanding of their roles within the wider clinical workforce, and led to more effective team working.

Staff feedback suggests the sessions are having positive benefits; they feel they are effective and enjoyable. Encouragingly staff have begun to take ownership of the sessions, suggesting topics for discussion. We have found that since introducing the ten-minutes sharing and learning sessions team morale has improved with staff telling us they feel included and valued. We plan to continue with the sharing and learning sessions with our intention being to build on our success. They are proving to be effective in ensuring our busy clinical workforce gains the knowledge and skills required to deliver high quality care to our patients.

\section{P-54 'RESPIRATORY REFRESHER': AN IN-HOUSE TEACHING PROGRAMME TO UPSKILL COMMUNITY NURSE SPECIALISTS}

Nicola Galliford, Laura Gordge. St Barnabas House Hospice, Worthing, UK

\subsection{6/bmjspcare-2019-HUKNC.78}

Introduction Ten of the community palliative care nurses working out of St Barnabas House Hospice in Worthing have previously attended a physical examination course. Some nurses have attended this very recently, but others less so. Informal feedback from these nurses suggested they lacked confidence performing examinations in their clinical practice and needed assistance in maintaining their skills. The inpatient unit often has stable patients with respiratory signs. Many of the doctors staffing the unit have an interest in teaching and have recently passed the clinical examination for membership of the Royal College of Physicians (PACES). A teaching programme was designed to share knowledge and address educational needs of the community team.

Methods Nine of the ten nurses signed up to a programme of 6 one-hour sessions in this pilot. Each session is limited to three nurses and is run by a ward doctor on the inpatient unit. The sessions are tailored to the attending community nurse specialists' needs and involve examining patients on the inpatient ward with respiratory signs. Attendees are required to fill in a short survey before attending the first session and after attending the last session to assess their subjective confidence levels in performing the respiratory examination and finding signs. The number of examinations performed during clinical practice per week before and after the course will also be measured as an assessment of behavioural change.

Results The project is currently in progress, results to be presented at the conference.

\section{P-55 THE VOICE BEHIND THE PALLIATIVE CARE ADVICE LINE: ENSURING KNOWLEDGE, SKILLS AND SUPPORT}

Sally Bailey. Dorothy House Hospice Care, Winsley, UK

\subsection{6/bmjspcare-2019-HUKNC.79}

Background National guidelines place an emphasis on patients, carers and health professionals being able to access specialist palliative care advice at all times (National Council for Palliative Care, 2015; Department of Health, 2008). The advice line enables this timely support and guidance. However, whilst running alongside the Inpatient Unit, the specific skills and knowledge required to handle calls can be overlooked (Carr, Lhussier \& Wilcockson, 2008).

Aims To support all Registered Nurses working on the Inpatient Unit to feel more confident and competent in taking advice line calls (Purc- Stephenson \& Thrasher, 2010) delivering up to date, safe and consistent advice, whilst enabling a culture of feedback and support.

Methods

- The development of advice line training following consultation with unit staff, feedback from other hospices and literature review, with sessions balancing specific telephone communication skills alongside clinical knowledge and practical elements (Yardley, Codling, Roberts, O’Donnell et al., 2009);

- The implementation of a new framework to help guide more complex calls - encouraging staff to think of completing a jigsaw puzzle without being able to see the picture on the box, and by putting together 'pieces' of information to guide the advice given by remembering to 'delve' and 'tell me more...';

- The commencement of regular feedback sessions enabling protected time for staff to 'offload', discuss difficult calls and for feedback and ongoing teaching from an Inpatient Unit Consultant or other palliative care professional;

- The development of an information pack to accompany training and an advice line resource folder for staff to reference during calls.

Conclusion Acknowledging that for some staff the advice line can be stressful, the implementation of training and support has had a very positive impact, with staff reporting a more confident approach when taking calls. The new resources have been welcomed and already proven useful, particularly when dealing with medication related calls. This work is already enabling the delivery of more consistent, confident and quality advice and in time this will undoubtedly increase caller satisfaction (Moscato, Valanis, Gullion, Tanner et al., 2007).

\section{P-56 TEACHING NURSES TO TEACH END OF LIFE CARE: CNSS' PERCEPTIONS OF THE IMPACT OF AN EDUCATIONAL INTERVENTION}

${ }^{1,2}$ Karen Groves, ${ }^{2}$ Barbara Jack, ${ }^{2}$ Mary O'Brien. ' Queenscourt Hospice, Southport, UK; ${ }^{2}$ Edge Hill University, Ormskirk, UK

\subsection{6/bmjspcare-2019-HUKNC.80}

Background Central to national and international policies is the need for generalist healthcare staff to have education in end-of-life care. Much end-of-life care education is provided by specialist nurses who often have no specific education development to prepare them to teach. To address this gap an 